\title{
«AUX ORDRES DE L’ÉPOUX OBÉIR, C'EST LE DROIT ESSENTIEL DES ÉPOUSES » : LE MARIAGE DANS LES ÉCRITS DE LA PRINCESSE FRANCISZKA URSZULA RADZIWIŁŁ (1705-1753)
}

\begin{abstract}
Judkowiak Barabara, "Aux ordres de l'époux obéir, c'est le droit essentiel des épouses 》 : le mariage dans les écrits de la princesse Franciszka Urszula Radziwitt (1705-1753) ["Obedience to one's husband is a wife's first duty" : the subject of marriage in the writings of Princess Franciszka Urszula Radziwiłł], Studia Romanica Posnaniensia, Adam Mickiewicz University Press, Poznań, vol. XXXVIII/1: 2011, pp. 67-79. ISBN 978-83-232-2279-8. ISSN 0137-2475. DOI 10.2478/v10123-011-0006-x.

This article deals with the issue of love, marriage and motherhood, which dominates the work of the first Polish female writer to be at once both a poetess and a dramatist. It offers a reconstruction of the biographical context that strongly influences many of Radziwiłłowa's utterances on this subject and sheds light on more general matters, such as the juridical and social situation of women in the Polish-Lithuanian Commonwealth.
\end{abstract}

Le fragment cité dans le titre du présent article et venant d'une lettre de la princesse à son époux est suivi de la conjonction « mais » qui met en cause l'importance du jugement à peine prononcé. Cette constatation sert d'une belle introduction à la présentation de l'œuvre de cette femme écrivain polonaise qui exprimait - quoique de manière discrète (pour ne pas dire timide) - ses doutes et une certaine objection quant à la norme patriarchale dominante. Elle était poétesse et la première femme dans la littérature polonaise qui se livrait à la création dramatique, destinée à être mise en scène par la troupe de théâtre de la cour de son siège familial à Nieśwież. Elle est née en Volynie, dans une famille polonisée des princes Wiśniowiecki. Durant une moitié de sa vie, elle était liée avec les biens de son mari en Biélorussie et en Lithuanie. Elle représentait les élites de la noblesse de la République des Deux Nations (nom officiel de l'état à l'époque) qui était, en fait, une fédération de plusieurs peuples. Aussi la princesse témoigne-t-elle, dans ses écrits, de l'enracinement européen de la culture des sphères de noblesse cosmopolites, mais, en plus, illustre-t-elle quelques spécificités législatives et idéologiques d'un grand pays aux confins de l'Europe (pour ne pas oublier ses mœurs). 
Il n'est pas difficile de constater que son œuvre concerne, en majorité, l'expérience féminine : questions d'amour, de mariage, de maternité. La princesse, auteur des Conseils salutaires à ma fille et traductrice des Points Réponse français pour les femmes (auxquels elle a ajouté sa Réponse adressée aux maris), est connue, pour certains lecteurs et chercheurs, grâce au contenu de ses écrits, commun pour toutes ses œuvres, qui se ressource dans ses expériences de vie d'une mère douce et attentive et d'une femme heureuse (voir p. ex. Kulesza-Woroniecka, 2003 : 633-634). Cette dernière observation sera nuancée dans la suite aussi bien pour son manque d'exactitude que son caractère trop général.

Sans doute, n'est-il pas insignifiant de constater que le mariage de Franciszka avec Michał Radziwiłł - indépendament du fait que la fille unique du prince Janusz Wiśniowiecki et de Teofila Leszczyńska était un bon parti ${ }^{1}$ - ne résultait pas tant d'un calcul économique ou politique des parents (il allait même à l'encontre de la mère apodictique du prince - dont le père était déjà mort à l'époque - Anna Radziwiłł) qu'il tenait à un coup de foudre de Michał qui avait déjà réfuté plusieurs candidates au mariage. En cette annéé 1725, Franciszka, âgée de vingt ans, s'est énamourée du prince Michał (aîné de deux ans à peine) qui - poussé par son oncle, frère de sa mère 2 - l'a découragée du projet de mariage avec le fils du commandant en chef adjoint de l'armée polonaise Stanisław Rzewuski - Seweryn. A l'époque, le projet de ce mariage était déjà avancé. Il vaut bien rappeler que le courage de s'opposer à la volonté de la famille était soutenu par le droit du couple de décider de la fondation de famille (droit appuyé, d'ailleurs, par l'Église), la suffisance de l'accord commun de la femme et de l'homme pour conclure le mariage, et la conviction de l'égalité des sexes avant de prendre une telle décision (Malinowska, 2008 : 18, 19, 27). Au début, le couple n'a pas reçu beaucoup d'aide de la part des parents. C'est pourquoi Franciszka et Michał avaient des problèmes financiers - quoique la perspective d'hériter, dans l'avenir, une grosse fortune restât toujours actuelle pour elle. La dot qu'elle a reçue et qui allait lui assurer une indépendance financière n'était pas suffisante. Michał, quant à lui, à côté des sœurs, avait un frère cadet, Hieronim. C'est sur ce dernier que la mère, après 1725 , a focalisé ses projets et ambitions. Ce n'est qu'après un certain temps que Michał est devenu un véritable chef de famille et a ramassé la majorité de biens familiaux. La

${ }^{1}$ Ce qui était le plus important pour les deux clans - même en dépit des parentés royales - était le fait que Franciszka, en tant qu'enfant unique de ses parents, allait hériter d'immenses biens fonciers. Or, généralement, on ne pouvait pas les apporter en dot. En plus, il est admis d'ordinaire qu'en Lithuanie, contrairement à la Pologne, la femme dotée d'une dot était même exclue de l'héritage familial (Popiołek 2003 : 212). Pourtant, la volonté des parents et les essais de Michał ont contribué finalement à la donation de la fortune - ce n'est qu'à trois mois avant le décès de Franciszka que la belle-mère âgée a fait le dernier legs de biens à Radziwiłł!

${ }^{2}$ Les jeunes, selon le droit canon, ont déjà atteint le soi-disant âge efficace autorisant le mariage avec l'accord des parents ou tuteurs (12 ans pour une fille, 14 ans pour un garçon) mais, selon le droit civil, il leur fallait attendre l'âge de 24 ans, exigé par ce droit (au moins dans la partie de la République appelée la Couronne de la Pologne) pour reprendre la capacité d'exercice, décider d'eux-mêmes et mettre fin à la tutelle paternelle. 
noblesse polonaise était obligée de conclure le régime de séparation des biens avant le mariage. Les parents de la princesse ont pris soin de l'avenir de leur fille déjà au moment de signer le contrat de mariage qui prévoyait le legs de toutes les seigneuries acquises par Radziwiłł à Franciszka après le décès du mari (conformément à la règle ius communicativum). En 1728, après avoir reçu les biens garantissant la dot de Franciszka de la part de la famille Wiśniowiecki, les époux ont signé un acte de donation réciproque et viager de tous leurs biens. Ils ont répété cet acte en 1737 en l'étendant à tous les biens mobiliers et immobiliers. La coutume polonaise de donation des biens par les époux assurait à celui qui survivait à la mort du conjoint l'usufruit viager des biens de ce dernier et réfutait la possibilité de la poursuite des droits par les héritiers (Malinowska 2008 : 50). Franciszka, après la mort de son père en 1741, a reçu tous ses biens fonciers mais, en même temps, a cédé le droit de gestion de ces biens (le pouvoir d'intervenir en justice le cas échéant compris) à son mari (Czamańska 2007 : 393, 395, 402). Quant à elle, la princesse, après la mort de son fils aîné (donné à l'éducation chez la grand-mère Anna), a limité ses devoirs d'assistances à son époux à cause de la maternité qui prenait de plus en plus de son temps. Elle s'est occupée de l'éducation du groupe des enfants de plus en plus nombreux, y compris les siens et ceux confiés à elle dans le but de les préparer à la vie de la cour qui, d'ailleurs, gagnait en considération au fur et à mesure que la situation économique du ménage s'améliorait. Le mariage de Franciszka et Michał était une union stable, traditionnelle, quant à la distribution de devoirs (elle accouche des enfants et les soigne, il gère la fortune; elle s'occupe du ménage, il s'adonne au service public), mais, grâce aux efforts de la princesse - comme l'estime Ilona Czamańska - « il était doté des signes de partenariat ». Les relations entre les époux qui s'adressaient (comme leur correspondance et le journal du prince en témoignent ${ }^{3}$ ) à eux-mêmes à l'aide de doux diminutifs, jusqu'à la fin de leur vie, étaient très bonnes - à tel point que le prince, même jusqu'en 1753, documentait les anniversaires de leur mariage en rendant grâce à la Providence pour un ami [sic!] si dévoué et impérissable, comme l'ancien polonais courtoisait les femmes à l'aide de la forme masculine. Quand Franciszka demandait de la discrétion à son mari, elle combattait pour l'affranchissement de la femme et la nouvelle vision de l'union intime et émotionnelle de deux personnes, indépendante d'interventions de famille : « Je te sollicite, mon cher Michel, la lecture de mes lettres par toi-même pour qu'elles ne passent pas ainsi d'une main à l'autre. C'est ni à mère ni à sœur, me semble-t-il, de connaitre la nature de relations entre la femme et le mari » (1731). De même avec une lettre à sa belle-mère où elle essaie de ménager la femme trompée d'un cousin : «Entre mari et femme, il est bien difficile d'intervenir » (1734).

En général, le mariage - quoique réussi - exposait Franciszka, à en croire ses œuvres et sa correspondance privée, aux souffrances liées à la norme culturelle qui

${ }^{3}$ Les manuscrits dans les départements IV et II des Archives des Radziwiłł (Archives Centrales des actes anciens de Varsovie). Il vaut bien, pourtant, prendre en compte que Franciszka signe ses lettres aussi avec des formules conventionnelles en tant que serviteur ou même... tabouret ! 
donnait à l'homme la liberté dans les contacts extra-conjugaux. Toute absence du mari qui prenait du temps et le manque de lettres de sa part causaient le désarroi de la princesse, les expressions de ses ennuis, les conjurations d'amour, les rappels des promesses d'avant le mariage et des engagements du serment nuptial mais avant tout la demande " montre à tout le monde que tu m'aimes d'amour quel qu'il soit » (Sajkowski, 1980 : 242). Des médisances et des intrigues s'y sont jointes. Il faut ajouter qu'il n'y avait pas de symétrie dans le respect de mœurs par les hommes nobles polonais et les dames dont les unions extra-conjugales et celles d'avant le mariage ne signifiaient nullement le déshonneur pour les messieurs mais seulement pour les femmes. C'est pourquoi le leitmotiv de ces poèmes sera la constatation que la princesse «honneur estime plus que la vie $»^{4}$. Cela était lié à la rigidité dont la noblesse polonaise faisait preuve en appliquant la question de la pureté de sang : dès 1578 (proclamation de la constitution De illegitima prole, affirmée contre les unions de personnes issues des états sociaux inégaux ${ }^{5}$ ) jusqu'en 1768, les enfants nés hors du mariage étaient exclus de l'héritage et de l'état noble (Malinowska $2008: 23-24$ ). En cas de prendre quelqu'un en flagrant délit d'adultère, la peine, dans la République des Deux Nations, était infligée aux représentants de deux sexes (en France, seulement aux femmes), pourtant ce n'est que le droit civil de la Couronne de la Pologne qui était libéral (quant à l'ampleur de la peine); tandis que la jurisdiction sévère du Grand-Duché de Lithuanie prévoyait la peine de mort, quoique, dans la pratique (influencée probablement par le droit canon plus libéral en la matière), on ne signait pas en général, quelques cas singuliers exemptés, les arrêts de mort (Malinowska $2008: 55,62$ ). Néanmoins, la jeune princesse écrivait à Radziwiłł : « je sais bien que les maris, absents de leurs femmes, s'adonnent souvent à des refraîchissements » et, avec de l'esprit, lui envoyait un melon, en lui demandant de « ne point chercher d'autre fraîcheur » lors du séjour hors de la maison.

C'est le thème de l'amour qui domine dans les seize drames de Franciszka U. Radziwiłł, en majorité des reprises des motifs connus ou des modifications des modèles différents. Ils illustrent le problème de manière originale et jamais univoque : de l'éloge de la virginité défendue au prix du martyre à l'époque des persécutions des chrétiens par Dioclétien (Tragédie de trois sœurs, Chionie, Agapè, Irène), par l'examen de la constance et de la fidélité d'un fiancé (Comédie de Partenie et Timant tirée du Grand Cyrus ou Artamène de $\mathrm{M}^{\mathrm{lle}}$ de Scudéry), à la Griselda polonaise obéissante et constante dans l'amour du mari qui multiplie cruellement les épreuves

${ }^{4}$ «Honneur plus estimé que la vie » - c'est cette citation du poème de la princesse que j'ai proposée pour le titre de mon compte-rendu plus large sur ce sujet ( «Conflit de nature et de culture dans l'œuvre de Franciszka U. Radziwiłł », dans : Les aristocraties en Europe du Moyen Âge à aujourd'hui. Actes du XVe Colloque organisé par l'Université Marc Bloch et UAM à Strasbourg, 9-10 octobre 2008 ; sous presse, Presses Universitaires de Strasbourg).

${ }^{5}$ Même si les parents se sont mariés dans la suite. La noblesse polonaise respectait le droit civil plus rigide que le décret d'après le concile de Trente traitant du sujet de mariage, appliqué en 1577 (Tametsi). 
de sa fidélité (Boccace Décaméron, nouvelle X, 10, adaptation polonaise sous le titre : Comédie de Cécile, ou Vertu éprouvée, ou L'or dans le feu ${ }^{6}$ ). Il ne faut pas oublier non plus les victoires ambiguës ${ }^{7}$ des femmes dans les récits orientaux, mises en pièces d'un acte pour le théâtre de l'opéra comique parisien déjà par un certain Pierre Gallet (Les coffres du marchand Banut ou Le double tour, c'est-à-dire Comédie de Laideur) ou l'ambivalence de l'image du mariage, tirée des farces, que présente Le médecin malgré lui de Molière avec la constatation, appuyée - à ce qu'il paraît - par l'autorité d'Aristote, concernant « la femme indocile, pire que le diable », avec les protestations de Martine contre Robert intervenant quand elle est battue par son mari (Sganarel) et, enfin, avec la déclaration de celle première, prononcée sous l'échafaud de celui dernier, qu'elle reste sa femme jusqu'à sa mort et qu'elle va « le disposer à cette mort cordialement ». Dans une pièce d'inspiration pastorale et mythologique sur Pâris, la princesse a fixé un dialogue d'époux dévoilant des relations typiques : Priam demande à Hécube de révéler la cause du désespoir et des sanglots mais l'impératif « Parle, c'est un ordre » est adouci par la persuaison : «N'es-tu pas ma femme unique [en son genre] et ma santé ». Sa réponse ardente « Oh ! mon mari, Oh ! mon roi, Oh ! mon maître, Oh ! ma vie !/ Je ne cache rien, je ne veux point mentir ! » masque le jeu mené par Hécube : dans les scènes suivantes, à la dérobée et contre la volonté de son époux, elle fait sauver la vie du fils qui vient de naître. Il n'est pas insignifiant, pour la question du mariage présentée par Radziwiłł, qu'à côté des amants ou des candidats au mariage, ce sont les parents qui apparaissent sur scène, témoignant de la prolongation de l'âge d'or des pères, représentant le pouvoir dans la famille. Ils sont accompagnés parfois des mères : la mauvaise mère de la fable, enviant à la fille sa beauté ( $L$ 'cuvre de la Providence divine) ou la mère douce et bienveillante mais naïve (Bogumiła, donnée à Griselda-Cécile parmi les personnages connus de la nouvelle de Boccace) - elle ne partage pas les mauvais pressentiments, d'ailleurs bien fondés, du père quant au destin de leur fille, gamine d'une pauvre famille, qui va devenir une dame au château. Dans la pièce L'amour parfait d'une dame, basée - elle aussi - sur une histoire du Décaméron - la femme d'Aristide lui demande de permettre à leurs filles d'obéir à la voix du cœur. Il faut souligner ici que les éléments didactiques et parénétiques dans l'œuvre de la princesse ne doivent point étonner : ce sont ses enfants (et ceux qu'on lui a confiés) qui se produisaient sur scène. Cela mettait en relief le contexte de l'union de deux personnes ainsi présenté et allait à l'encontre des idées d'émancipation, exprimées par

${ }^{6}$ Pour plus d'informations sur cette pièce, voir mon intervention lors du $17^{\mathrm{e}}$ Colloque International La représentation du feu sur les scènes du théâtre et de l'opéra organisé par la Société Internationale d'Histoire Comparée du Théâtre, de l'Opéra et du Ballet, et par l'Université de Messine, à Montalbano Elicona, 18 - 22 septembre 2009 (à l'impression).

${ }^{7}$ C'est ainsi que le veut Dobrochna Ratajczakowa - à cause de la mise en bouche d'Arlequin de l'éloge triomphal avant le jugement sur l'héroïne (Comédie des éclairés 1993 : 26). Il semble pourtant que ce personnage de moralité douteuse n'est pas capable de voiler les faits : Aruja du Fripon en piège résume la question par l'aveu, devant son mari, de ce que les spectateurs déjà savent, c'est-à-dire, de la fidélité gardée par elle durant toutes ces aventures. 
Radziwiłł dans d'autres situations ${ }^{8}$. En Pologne, comme en Europe, on se mariait en présence des parents ce qui tenait à assurer la situation financière de la progéniture. Les jeunes amantes des pièces de la princesse Radziwiłł prennent en compte l'avis de leurs parents, leur déclarent l'obéissance dans le choix du candidat au mariage comme Tymaretka dans Comédie de Sésotris (dont l' intrigue, de nouveau, est tirée d'Artamène) ou Filida dans la comédie L'amour parfait... Dans l'acte IV, les chevaliers enlèvent les demoiselles qui les avaient réjetés (témoignant ainsi de la pratique du rapt, en vigueur au XVII ${ }^{\mathrm{e}}$ siècle aussi bien en France qu'en Pologne) cependant, l'héroïne principale, Filida, se rebelle et sollicite l'accord du père pour son mariage.

Et pourtant, le motif préféré de la princesse, lui permettant de discuter les règles de mœurs et les lois, était la liberté d'un individu. Toutes les filles, sollicitées par les amants, font attention à la perte de la liberté. En général, « le joug de l'obéissance » les effraie. Cela s'explique aussi bien par de nouvelles idées dans le domaine de sentiments (nous allons en reparler dans la suite), propices au libre choix fait par des individus dotés d'une conscience de leur singularité (ce qui serait un héritage de l'époque baroque) que par un trait polonais spécifique (soutenu par la culture politique dominante à l'époque et le régime républicain ${ }^{9}$ ). Il vaut bien rappeler que c'est une femme avec de l'expérience dans la vie qui écrivait ces pièces (le théâtre fonctionnait à Nieśwież dès 1746 , date de son mariage, tandis que l'œuvre poétique de la princesse, a commencé une vingtaine d'années auparavant).

Le thème de l'amour conjugal est apparu pour la première fois dans ses lettres, parfois rimées, adressées au prince que Franciszka venait d'épouser et dont les absences, de plus en plus longues et fréquentes, à cause du service public, causaient beaucoup de peine à la jeune épouse, esseulée et toujours languissante (Mycielski, 1889 ; Judkowiak, 1992). Il est repris de même dans l'œuvre poétique, personnelle et plus tardive, comme Poèmes et plaintes sur le mariage et regrets dont la dimension plus intime fait prendre l'autobiographisme pour une hipothèse nécessaire (Ziomek, $1975)^{10}$. Cette partie de l'œuvre de l'auteur restait en forme des manuscrits copiés

${ }^{8}$ Une des filles de la princesse a pourtant bien tiré les conséquences du climat de l'éducation que sa mère lui avait assuré : elle réalisait le scénario du mariage conclu d'amour et de libre décision contre les calculs dynastiques liés aux alliances possibles entre les princes européens ou les grands seigneurs polonais : elle s'est enfuie avec son amant, du rang social inférieur (chef de la garde de château, Morawski), a obligé son frère Karol Stanisław, son tuteur après la mort des parents, d'accepter cette mésalliance scandaleuse (Sajkowski, 1981 : 314-343 ; Taylor-Terlecka, 2003).

9 Radziwiłł a inclu les références à la liberté de la noblesse, valeur très appréciée par les gentilshommes et principe du régime gardé du $\mathrm{XVI}^{\mathrm{e}}$ siècle et influançant la vie quotidienne, dans Les conseils salutaires à ma fille et dans la législation de Solonia dans la pièce L'amour né dans les yeux (encore une fois tirée d'Artamène).

${ }^{10}$ La constation connue de M. Foucault sur l'Européen comme « animal confessant » et sur « le discours de vérité » demandant sa révélation selon le modèle de la confession (surtout la vérité des sentiments opposés à la régle imposée, p.ex. concernant le mariage) soutient cette hypothèse dans le cas de la lyrique de l'aveu intime. Voir M. Foucault, Histoire de la sexualité (Paris : Gallimard 
seulement pour la famille et quelques-uns, après sa mort, ont rejoint d'autres brouillons dans une poubelle à papiers venant de la bibliothèque. On trouve, dans les aveux lyriques de Radziwiłł, des traces de déceptions amoureuses passagères (« Pour jouir de l'amour non équivoque, il faut qu'il soit réciproque »), de soupçons, de jalousie, de peine de suivre le serment de fidélité toute seule et de dissimulation devant les intriguants de la cour. Le droit libre d'aimer - qui fait partie de ce recueil - est en opposition, seulement en apparence, avec les conseils adressés $\grave{A}$ la dame volage qui, au lieu d'attendre patiemment le seul candidat au mariage, attire une foule d'amants galants. La conception du mariage, exprimée en langage de droit (ligue, décret), est liée ici, significativement, avec une argumentation de nature morale et religieuse (vertu, c'est-à-dire fidélité, soumise à la volonté divine, à savoir la monogamie) et avec la motivation qui relève de la raison contrôlant les sentiments et leur authenticité : n'est-ce pas que « l'amour n'accepte point les cœurs partagés » et qui met en lumière les intrigues des galants. La recherche de la stabilité émotionnelle, exprimée à peine dans celui et d'autres textes, est opposée au flirt. Ce Droit libre d'aimer, mentionné plus haut, est donné par la princesse comme « loi sur l'amour » pour les jeunes ${ }^{11}$. Mais cette loi naturelle, permettant de suivre ses émotions et « les instincts cachés », est en conflit avec la loi divine qui sert d'appui, vraisemblablement, à l'harmonie résultant de l'ordre social dominant. Or, la loi d'origine divine est soutenue par les notions employées par la princesse (appelées par les sociologues régulateurs sociaux) : raison et honneur faisant oublier « orages du cœur » et « soigner les blessures d'amour ». L'œuvre finit par un soupir et une prière «Ah, quelle loi impitoyable! Dieu, change la nature ou ce droit lamentable ! ». À l'aide des notions du domaine de droit, la princesse traite d'un problème plus large de l'anthropologie, de l'éthique et de la pédagogie européennes : conflit de la nature et de la culture. Même si elle acceptait de maîtriser le côté biologique de l'individu humain en s'appuyant sur les catégories comme : raison, modération, vertu, courage, honneur, gloire, elle ne permettait pas d'enfermer les femmes seulement dans la nature (et l'infériorité aux hommes).

En plus, ces poèmes traduisent la déception féminine de la vilenie des hommes qui ne s'abstiennent pas de flirter sous le prétexte de conclure un contrat ou d'assurer l'alliance du point de vue formel : « au commencement, rien que des paroles, des serments, des obligations et des iurament [selon le droit polonais ancien, affirmation à valeur d'une preuve] » mais après « une minute, l'espoir culbute » (Chant). La princesse formule ces accusations grâce à sa " pratique », " ayant éprouvé des infidélités incomptables ». La femme est confrontée au sentiment de l'abus émotionnel et social, à la vérité sur l'insincérité des affects dont les promesses l'ont bercée et « à l'abus [...]

1976-1984). La princesse, consciente du caractère bouleversant et stigmatisant de ses confidences, les a enfermés dans un tiroir.

${ }^{11}$ La suite de cette discussion dans la pièce L'amour parfait d'une dame : la sœur aînée raille, avec de l'ironie, les scrupules de l'héroïne : « Obéir à son mari ce n'est pas grand-chose, quelle loi nous y forcerait? [...] On peut suivre sa lubie, mais en cachette ». 
à l'atteinte à l'honneur et à sa gloire ». L'intériorisation par les femmes de ces deux dernières valeurs et des interdits et des ordres qui les accompagnaient - renforcés par le système d'éducation propice à l'institution du mariage ${ }^{12}$ - menait, notamment dans les sphères de la cour, aux conflits intérieurs, attisés par la practique de mœurs (beaucoup plus libérale pour les hommes), invitant au flirt et permettant des histoires d'amour dans l'ambiance du rococo. Ayant remarqué que « le monde a changé de nature ces jours-là », la princesse exprime sa mélancholie et rappelle le passé auquel « la mode toute différente » et «l'hypocrisie de mœurs » s'opposent, tant critiquées par elle :

Pour jamais révolus ces temps heureux,

Où l'homme moins parlait pour plus aimer $[\ldots]$

Quiconque usait d'un doux langage,

Se trouvait enclos dans une cage (inc. "Qui sait lire dans les caractères...»).

À partir du XVII ${ }^{\mathrm{e}}$ siècle, les dames, dans la culture française et, comme on le voit, dans les zones de son influence, demandaient l'équilibre d'énergie et d'initiative dans le mariage pour passer de l'emprise du mari sur la femme au partenariat des époux. De plus en plus souvent au XVIII ${ }^{e}$ siècle, quand l'individu humain devient un être instable, en train du développement, son amour, quant à lui, devient plus stable (autrefois changeant, contrairement à la stabilité du caractère déterminé par le tempérament) et, ainsi défini, peut servir de base au mariage (Luhmann, 2003 : 123). Il semble que cette attente est déjà formulée dans les lettres en vers que Franciszka adresse au prince Radziwiłł. L'appel à la fidélité accentue la possibilité de stabiliser leur union. Cela est bien visible dans les titres des lettres : «Le prince auquel je suis lié d'un amour nous unissant dans le mariage » (Lettre I, vers 1-2), dans le nom qu'elle attribue à son mari : « la seule consolation de mon âme» (I, 35), mais aussi dans le fait de souligner l'authenticité du sentiment indépendant des liaisons conjugales : «je t'aime non seulement par le serment/ [...] Même en dehors de l'hymen, / Tu serais ma joie certaine » (III, 11, 13-14). En échange de son amour, elle attend que celui de son mari pour elle soit exclusif ( «il n'y a pas d'amour sans reconnaissance» III, 71). C'est une vision de l'union conjugale inséparable même dans l'éternité (III 59, 67-68) qui permet de réfuter des reproches : « l'hymen n'est jamais une douleur/ Quand il unit corps, raison, goût et cœur » (III, 57-58).

Ce qui attire l'attention dans les manuscrits de la princesse Radziwiłł ce sont les pages de la prose en français Du mariage et Des devoirs réciproques de l'homme et de la femme dont les sources d'inspiration et les modèles restent jusqu'aujourd'hui méconnus ${ }^{13}$. Quelques chercheurs, suggérés par le titre donné à tout ce recueil, prennent

12 « N'est-il point atteinte à l'honneur/ d'aimer dans le mariage » chante Klorysa dans L'amour plaisantant (Radziwiłłowa, 1754 : I, 7). Dans le cadre d'une union accepté par la société - conformément à la formule ecclesiastique matris monium, c'est-à-dire défendant les droits de la mère c'est la famille, base de l'organisation de la société, qui se crée.

${ }^{13}$ Gardés dans la Bibliothèque des Czartoryski (côte 2268, pp. 71-85 et 91-106). 
ces textes pour des écrits originaux de la princesse ce qui représente un certain abus (Rarot 2006). Notre attitude est plus prudente - au fur et à mesure de la découverte des œuvres des auteurs français dans ce brouillon de la princesse, nous sommes enclins à constater que l'ensemble est un recueil copié par elle. Les deux traités sont marqués par la présence du sujet masculin qui présente le point de vue masculin sur le mariage. Bien que l'homme soit ici doté de la position privilégié, grâce à l'autorité et aux capacités lui accordées par Dieu, il est souligné que sa souveraineté doit relever de l'amour et de la justice. L'époux ne peut pas traiter sa femme en esclave, bonne, ou nourrice mais en personne du même rang que le sien. L'épouse, quant à elle, ne peut pas négliger les divertissements diverses pour que son mari ne s'ennuie pas à cause de la monotonie (p. ex. Bogucka 1998 : 146 ; Sajkowski, 1980 : 259-260). Quoi qu'il en soit, le choix des textes sur le mariage et leur continu (le second met en relief la mutualité des devoirs par son titre même) sont un indice pour un chercheur interprétant l'œuvre littéraire de la princesse. Or, le pas suivant sera pour elle d'accompagner sa traduction des Points... français d'une Réponse de l'épouse à son mari ${ }^{14}$. Les points français prônent l'idée que la femme doit adoucir, par son amour, " le doux joug » de l'union sacrée à son époux, à savoir ne pas fréquenter, contrairement aux mœurs à la mode, d'autres hommes. La princesse y répond en soulignant que les promesses sont réciproques et que « le mari doit garder le même engagement [fidélité] » ${ }^{15}$. Ainsi, les engagemnts symétriques sont énumérés : " de même avec le mari », fondés sur l'idée que « c'est la fidélité réciproque que nous avons jurée ». Donc, même ici et conformément à ce que nous avons constaté plus haut, les ordres extérieures sont argumentés par la nécessité de choix moral, de maîtrise des pulsions intérieures (Judkowiak, 1992 : 65). Malheureusement, l'original français de ces douze Points de six vers reste toujours non identifié...

Dès le XVII ${ }^{\mathrm{e}}$ siècle, l'investigation psychologique, indispensable à l'affirmation du libre choix, devenait de plus en plus importante dans la culture européenne ; à partir de 1700, on comprenait l'amour plutôt comme sympathie ce qui le rendait similaire à l'amitié et contribuait aussi à mettre fin au manque de symétrie du pouvoir dans les familles. Aussi découvrait-on la femme en tant qu'individu humain, capable de retrouver sa place et sa condition morale dans le mariage. Cette prise de conscience démolissait les hiérarchies dans le couple conjugal (Luhmann, 2003 : 124). Quelles que soient donc les garanties incessantes de la soumission de la princesse à son époux (qui ne relèvaient pas que de la coquetterie), un tel verbage ne doit pas nous tromper. La correspondance de Franciszka est marquée par la combinaison de soumission et de

${ }^{14}$ Les manuscrits de la Bibliothèque de l'Académie Polonaise des Sciences à Kórnik, côte 1604, pp. 16-23 et 19-22 et de la Bibliothèque des Czartoryski, côte 2332, pp. 22-25 et 25-28. Dans le titre de la Réponse, on a souligné : composée par moi-même.

${ }^{15}$ La question est reprise aussi dans la pièce L'amour plaisantant (1746) : les filles frivoles rejettent les conseils du père (dont l'idéal de la femme est lié, selon les chercheurs, aux idées d'Arnolphe de Molière). Elles réfutent ses arguemnts à la dérobée, en rappelant qu'il flirtait avec d'autres femmes dans sa jeunesse même après le mariage. 
tendresse comme dans le fragment qui suit : « Et pourtant, je vais très bien. Toutefois, à un serviteur, il n'y a qu'un seul être qui manque, son maîre. Alors, c'est à toi qui es la plus grande joie de ma vie que j'adresse cet appel : viens chez celle qui te jure son amour fidèle et impérissable jusqu'à la fin de sa vie ». Dans le Poème à sa Majesté le Prince, Franciszka Urszula écrit p. ex. que, pour apaiser une angoisse à lui, elle a peur d' " aigrir son doux maître » mais ajoute aussi qu'elle tient à son bien-être et à sa gentillesse. Inquiète et languissante à cause de l'absence de Michał, elle l'appelle souverain de son cœur et croit convenant de l'assurer : « je tiendrai ma promesse avec engagament ", " jusqu'à la fin de mes jours, ce n'est que toi - mon amour ». Cependant, ce ne sont pas que des déclarations d'obéissance, de soumission et de fidélité mais surtout d'affect qui attend une réponse, une consolation « dans l'amour réciproque » et le retour vite du mari éloigné. La princesse ne s'abstiendra non plus de mettre en cause le sentiment de pouvoir - nuisant à la vie de couple - qui résulte de l'encadrement du mariage par le droit : «À cause de l'excès de pouvoir rétrograde/ Même l'amour sacré des époux se dégrade ». Cette amère constatation sera reprise dans les poèmes-plaintes conjugales, renforcée par la déception amoureuse (« Moyennant mon amour ce n'est que le dédain que je reçois ») et par la désillusion : «l'époux fuit sa femme » et «l'adultère l'a emporté sur l'hymen».

Pourtant, les œuvres de circonstances, comme félicitation à l'occasion du mariage (Poèmes sur le mariage de Józef Potocki, 1732), expriment les idées générales et les lieux communs concernant le mariage. Cependant, au lieu de lier l'hymen et l'amour justement aux loci communes, Radziwiłł, poussée par les conventions en vogue dans le milieu noble réglant les discours et les toasts de mariage, a joint l'éloge des nouveaux mariés et l'augure sur leur vie future aux mérites de leurs ancêtres et aux concepts héraldiques. L'auteur était plus intéressée par la fécondité du couple que par la situation sentimentale de la jeune fiancée Ludwika Mniszchówna, donnée au riche veuf qui pourrait aussi bien être son père... Il est significatif qu'elle passe sous silence le fait donnant du piquant à la situation, à savoir le projet du mariage de Ludwika avec le fils de Józef qui, une fois devenu veuf, a repris la place promise à son enfant (Popiołek, 2003 : 203). Le mari allait assurer le bonheur de sa deuxième épouse, outre la fortune et le rang social, par le même estime dont il faisait preuve à l'égard de la première.

Quant aux Points pour Arlequin ${ }^{16}$, auquel on conseille l'agitation continue autour de sa femme paresseuse, ils constituent une version italianisée (ou tirée de la littérature italienne - ce qui n'est pas encore tranché) du personnage de mari-bouffon, connu dans les parodies bourgeoises ou plébéiennes des " lois » ou règlements. Ils mettaient à rebours les relations traditionnelles entre les sexes. Le monde ainsi créé (où l'homme était serviteur de sa femme) amusait par le détournement de rôles, carnavalesque et - partant - temporaire. Ces œuvres rejoignent donc, dans l'histoire de

${ }^{16}$ Copie parmi les poèmes de la princesse dans les Archives à Mińsk. Je remercie la docteur Natalia Rusiecka et le professeur Sergiusz Kowaliowow de me l'avoir rendue accessible. 
la littérature officielle et masculine, le genre de satire antiféminine qui montraient les aspirations incongruës, mais somme toute inoffensives et irréelles, des représentantes $\mathrm{du}$ « deuxième » sexe. Dans un manuscrit féminin, elles deviennent l'expression des rêves compensateurs, interprétés avec de la distance ludique.

En conclusion, il convient de suggérer la nécessité de poursuivre les recherches sur l'influence de l'œuvre de Madeleine de Scudéry sur l'écriture de l'auteur polonaise. Étant donné la dépendance de trois pièces de la princesse des nouvelles tirées du Grand Cyrus, il est presque indubitable qu'elle avait aussi lu le livre premier de la partie VII du roman où, durant une discussion sur le mariage, les arguments d'une ennemie intransigeante de tristes liaisons se font la voie : même si les jeunes époux s'aiment véritablement, cet état n'est pas donné une fois pour toutes. Une épouse soumise à son mari perd progressivement sa santé, sa beauté et la jeunesse. En plus, elle est confrontée à la jalousie du mari ou en éprouve elle-même. Elise condamne tous ceux qui soutiennent que le mariage ou le cloître sont les deux seules voies possibles pour une fille. Elle réfute l'opinion selon laquelle la vertu, pour être maintenue, demande soit un mari soit une haute muraille. Quant au bonheur dans le mariage, elle argumente que l'amour, la jeunesse et la fortune sont capables de le garantir mais seulement temporairement - car toute union devient, au fur et à mesure, malheureuse. Il y a plusieurs conditions nécessaires au bonheur : sauf l'amour réciproque, l'hônneteté du mari, disposant des biens égaux à sa position, évitant des bizzareries, la jalousie et l'avarice. L'épouse est censée pourtant connaître ses centres d'intérêts, égaler ses ambitions, se soumettre entièrement à ses humeurs, lui être docile sans broncher jusqu'au point de renoncer à sa liberté dans les choses les plus difficiles - elle n'est même pas maître de sa personne. Chargée du devoir de tenir le ménage, elle court le risque de perdre la santé et la beauté en même temps avant que sa jeunesse ne passe. Il faut, donc, beaucoup de courage audacieux pour se décider d'une traite à une telle union, constate Elise $^{17}$. Cette vision réflèterait simultanément les plaintes éternelles des hommes sur la vie avec une feme qui n'augmente que des problèmes ou, dans des versions misogynes moins raffinées, l'aversion des "philosophes» contre le mariage : " guerre éternelle », " ridiculisation » et honte aux représentants $\mathrm{du}$ « premier sexe ». Certains échos des arguments d'Elise retentissent dans les interventions des héroïnes de la princesse Radziwiłł.

Pour clore définitivement ces quelques remarques sur le mariage dans l'œuvre de la femme écrivain polonaise, je citerai la suite de la lettre dont j'ai tirée le titre du présent article :

Obéir aux ordres des époux, c'est le droit essentiel des épouses. Ce n'est pas qu'à cause du pouvoir de Votre Majesté l'époux et le bienfaiteur, mais par l'amour pour vous, insondable et doux, que je suis prête à donner satisfaction à tous vos souhaits [et il voulait qu'elle cessât d'allaiter leur petite fille]. Mais, mon cher époux, veuille prendre en compte que le temps difficile où les dents de Tola se mettent à percer vient de commencer. Mon cher

\footnotetext{
17 Texte mis en ligne par www.gallica.bnf.fr.
} 
Michał, ne m'accable surtout point. Puisque c'est ton sang que je vénère en elle. C'est un signe que je ne veux aimer que vous-même et ce qui de vous est conçu [...] chaque goutte découlant sur les lèvres de la petite me rappelle cette vérité que je ne dois qu'aimer l'un pour l'autre.

Voici les arguments maternels dont Franciszka Radziwiłł, à l'époque des nourrices qu'on employait souvent dans les sphères nobles, essayait de persuader son mari. Or, la femme est, le plus souvent, aussi une mère et il est bien difficile de séparer ce fait des relations conjugales difficiles qui relèvent non seulement du droit, des mœurs et de la vie sentimentale.

\section{BIBLIOGRAPHIE}

Radziwiłłowa Franciszka Urszula z Wiśniowieckich (1754). Komedyje i tragedyje przednio dowcipnym wynalazkiem, wybornym wiersza kształtem, bujnościa rzeczy i poważnymi przykładami znamienite, b.m. dr. [Żółkiew].

Teatr Urszuli Radziwiłlowej (1961). Oprac. K. Wierzbicka, wstęp J. Krzyżanowski, Warszawa : Państwowy Instytut Wydawniczy [reedycja 4 dramatów: Opatrzności Boskiej dzieła, Złota w ogniu, Niecnoty w sidłach, Komedii wytwornych $i$ śmiesznych dziwaczek].

Bogucka M. (1998). Białogłowa w dawnej Polsce. Kobieta w społeczeństwie polskim XVI - XVIII wieku na tle porównawczym. Warszawa: Wydawnictwo Trio.

Czamańska I. (2007). Franciszka Urszula, Dans I. Czamańska, Wiśniowieccy. Monografia rodu (pp. 391-404). Poznań : Wydawnictwo Poznańskie.

Judkowiak B. (1992). Słowo inscenizowane. O Franciszce Urszuli Radziwillowej-poetce. Poznań : Wydawnictwo WiS.

Kulesza-Woroniecka I. (2003). Aktywność arystokratek w XVIII wieku. Dans Władza i prestiż. Magnateria Rzeczypospolitej w XVI-XVIII wieku, dir. J. Urbanowicz et al. Białystok : Wydawnictwo Uniwersyteckie.

Luhmann N. (2003). Semantyka miłości. O kodowaniu intymności, trad. J. Łoziński, Warszawa : Wydawnictwo Naukowe Scholar.

Malinowska M. (2008). Sytuacja kobiety w siedemnastowiecznej Francji i Polsce. Warszawa : Wydawnictwa Uniwersytetu Warszawskiego.

Mycielski J. (1882). Matka Księcia Panie Kochanku, Przeglad Polski, XVI, 369-380.

Popiołek B. (2003). Kobiecy świat w czasach Augusta II. Studia nad mentalnościa kobiet z kręgów szlacheckich. Kraków : Wydawnictwo Naukowe Akademii Pedagogicznej.

Rarot H. (2006). Traktaty o życiu szczęśliwym według Franciszki Urszuli Radziwiłłowej. Dans Праиы кафедры гісторыі беларускае літаратуры Белдзяржуніверсітета (pp. 158-166), vol. 7. Mińsk, WTAA Prawa i ekanomika [en ligne www.khblit.narod.ru].

Ratajczakowa D. (1993). Komedia oświeconych. Warszawa : Wydawnictwo Naukowe PWN.

Sajkowski A. (1981). Staropolska miłość. Z dawnych listów i pamiętników. Poznań : Wydawnictwo Poznańskie ; voir chapitres IX et XII. 
Taylor-Terlecka N. (2008). Kwartet na trąby - krnąbrna Teofila z rodziną w tle. Dans Kobieta epok dawnych w literaturze, kulturze i społeczeństwie (pp. 348-347) dir. I. Maciejewska, K. Stasiewicz. Olsztyn : Wydawnictwo Littera.

Ziomek J. (1975). Autobiografizm jako hipoteza konieczna. (Treny Jana Kochanowskiego). Dans Biografia-geografia - kultura literacka, (pp. 215-243) dir. J. Ziomek, J. Sławiński. Wrocław : Zakład Narodowy im. Ossolińskich; przedr. w J. Ziomek (1980). Powinowactwa literatury. Warszawa : PWN. 